\title{
Fatty acids-stress attenuates gluconeogenesis induction and glucose production in primary hepatocytes
}

\author{
Noga Budick-Harmelin ${ }^{1}$, Sarit Anavi ${ }^{1}$, Zecharia Madar ${ }^{1}$ and Oren Tirosh ${ }^{1,2^{*}}$
}

\begin{abstract}
Background: Hepatic gluconeogenesis tightly controls blood glucose levels in healthy individuals, yet disorders of fatty acids (FAs) oxidation are characterized by hypoglycemia. We studied the ability of free-FAs to directly inhibit gluconeogenesis, as a novel mechanism that elucidates the hypoglycemic effect of FAs oxidation defects.

Methods: Primary rat hepatocytes were pre-treated with FAs prior to gluconeogenic stimuli with glucagon or dexamethasone and CAMP.

Results: Pre-treatment with 1 mM FAs (mixture of 2:1 oleate:palmitate) for 1 hour prior to gluconeogenic induction, significantly decreases the induced expression of the gluconeogenic genes phosphoenolpyruvate carboxykinase (PEPCK) and glucose-6-phosphatase (G6pase) as well as the induced glucose production by the cells. The inhibitory effect of FAs upon gluconeogenesis is abolished when pre-treatment is elongated to 18 hours, allowing clearance of FAs into triglycerides by the cells. Replacement of palmitate with the non-metabolic fatty acid 2-bromopalmitate inhibits esterification of FAs into triglycerides. Accordingly, the increased exposure to unesterified-FAs allows their inhibitory effect to be extended even when pre-treatment is elongated to 18 hours. Similar changes were caused by FAs to the induction of peroxisome-proliferator-activated receptor- $y$ coactivator 1a (PGC1a) expression, indicating this transcriptional coactivator as the mediating link of the effect. This inhibitory effect of FAs upon gluconeogenic induction is shown to involve reduced activation of CAMP response element-binding (CREB) transcription factor.
\end{abstract}

Conclusions: The present results demonstrate that free-FAs directly inhibit the induced gluconeogenic response in hepatocytes. Hence, high levels of free-FAs may attenuate hepatic gluconeogenesis, and liver glucose output.

Keywords: Hypoglycemia, Liver, $\beta$-oxidation disorders

\section{Background}

The liver is a major site of systemic metabolic regulation. Pathways of synthesis and degradation of lipids and carbohydrates are controlled by complex interactions in the hepatocytes [1]. Induction of hepatic gluconeogenesis (GNG) promotes the synthesis and release of glucose into the blood, in order to maintain fasting normoglycemia among healthy individuals [2]. However, impaired glucose homeostasis, which leads to fasting-

\footnotetext{
* Correspondence: otirosh@agri.huji.ac.il

${ }^{1}$ School of Nutritional Sciences, Institute of Biochemistry, Food Science and Nutrition, The Robert H. Smith Faculty of Agriculture, Food and Environment, The Hebrew University of Jerusalem, Rehovot, 76100, Israel

${ }^{2}$ The Hebrew University of Jerusalem, P.O. Box 12, Rehovot, 76100, Israel
}

related hypoglycemia, is a prominent pathological feature of different fatty acids (FAs) oxidation disorders [3].

The main cellular site for oxidation of FAs is the mitochondria [4], where they are degraded in a cyclic process of $\beta$-oxidation into acetyl-CoA units. Disorders of mitochondrial FAs oxidation comprise a group of at least a dozen inherited defects of distinct enzyme or transporter deficiencies [5]. These diseases vary in their symptoms and severity, yet hypoglycemia is one major clinical sign in all FAs oxidation defects [6].

The pathogenic mechanisms leading to hypoglycemia in FAs oxidation defects have only partially been elucidated [7]. The observed disruption in carbohydrate management may result from combined effect of two 
processes. First, hypoglycemia can occur due to enhanced peripheral glucose uptake as an outcome of livers' failure to produce ketone bodies [3]. Second, hypoglycemic response might reflect reduced GNG. In this regard, several different factors have been suggested to contribute to an insufficient hepatic glucose production among patients. Those include lack of acetyl-CoA which is a product of FAs oxidation and serves as allosteric activator of pyruvate carboxylase, high levels of non-esterified acyl-CoA esters that hamper activation of pyruvate carboxylase, and low levels of ATP and reducing equivalents (NADH) necessary for GNG $[8,9]$.

The current study shades new light on the mechanistic basis of limited gluconeogenic capacity among patients with defects in FAs oxidation. It is demonstrated, for the first time, that FAs directly attenuate the induction of gluconeogenic programme in isolated primary hepatocytes and that removal of FAs restores the gluconeogenic response. Our findings indicate that free-FAs may be involved in inhibition of GNG induction, mediating hypoglycemia in FAs oxidation disorders.

\section{Results}

\section{Free-FAs treatment of primary hepatocytes}

Rapid uptake of FAs by cultured primary hepatocytes is indicated by TLC separation of cellular lipid extracts. Eminent increase in cellular FAs amounts is noticed after 1 hour exposure to FAs (Figure 1A). Longer exposure to free-FAs allows their esterification into triglycerides (TGs) by the cells, as indicated by time-dependent cellular accumulation with TGs (Figure $1 \mathrm{~B}$ and 1C). Indeed, as exposure of cells to FAs is elongated beyond 1 hour, free-FAs amounts decrease back to control levels (Figure $1 \mathrm{~A}$ and $1 \mathrm{C}$ ), parallel to the increase in TGs amounts.

As seen also by FACS analysis after Nile-Red staining (Figure 1D), TGs accumulation is not evident after exposure to FAs for 1 hour. Yet, as expected, massive increase in cellular TGs is noticed in cells after 18 hours exposure to free-FAs (Figure 1D). Similar results were observed when Nile-Red stained cells were examined under fluorescence microscopy (Figure 1E).

Replacement of palmitate acid with the non-metabolic FA 2-bromopalmitate (FAs-Br) markedly prevented the TGs accumulation observed after 18 hours (Figure 1D and $1 \mathrm{E}$, right lower panel). 2-bromopalmitate is not esterified into neutral lipids and inhibits esterification of endogenous FAs [10] and therefore prevents TGs synthesis in the cells.

\section{Free-FAs inhibit gluconeogenesis induction}

Based on the results presented in Figure 1, the effect of free-FAs on gluconeogenesis induction was investigated in primary hepatocytes by pre-treatment with FAs for 1 hour, prior to gluconeogenic stimuli.

Treatment of the cells with glucagon and cAMP for 6 hours induces a 16-fold increase in phosphoenolpyruvate carboxykinase (PEPCK) mRNA expression level (Figure 2A, left). PEPCK expression level is of main importance in gluconeogenesis regulation, and therefore is expected to be increased by this stimulus. As seen in Figure 2A (left), pre-treatment with FAs for 1 hour, prior to glucagon and cAMP, significantly decreases this PEPCK induction. However, 18 hours pre-treatment with FAs, which enables their esterification into TGs, has no effect on PEPCK induction by glucagon and cAMP. Accordingly, when cells are exposed to FAs-Br, which inhibits TGs synthesis and therefore allows more free-FAs, PEPCK induction is inhibited, even after 18 hours of pre-treatment.

Similar effects of free-FAs on PEPCK expression levels are seen when gluconeogenesis is induced by dexamethasone and cAMP (Figure 2B, left).

In addition, pre-treatment with FAs for 1 hour prior to glucagon and cAMP abolishes the induction of glucose-6-phosphatase (G6pase) expression in primary hepatocytes (Figure 2A, right). Like PEPCK, G6pase participates in gluconeogenic rate control in the liver. This inhibitory effect is also observed when cells are pretreated with FAs-Br, but not with FAs, for 18 hours before gluconeogenic induction (Figure 2A, right). Likewise, when induced by dexamethasone and cAMP, G6pase expression is effected by the pre-treatments in the same manner (Figure 2B, right).

As seen in Figure $2 \mathrm{C}$ and D, none of the treatments altered the expression of carnitine palmitoyltransferase 1 (CPT1), the rate-limiting enzyme in long-chain FAs $\beta$-oxidation, and of glycerol-3-phosphate acyltransferase (GPAT), the rate-limiting enzyme in TGs synthesis.

Since both PEPCK and G6pase are rate-limiting enzymes in the gluconeogenic pathway, the inhibition of their induction represent the ability of free-FAs to inhibit induction of GNG in hepatocytes. The inhibition of GNG by free-FAs was observed as pre-treatment with free-FAs for 1 hour, but not for 18 hours, significantly inhibited glucose production by hepatocytes, when induced by glucagon and cAMP (Figure 3A) or by dexamethasone and cAMP (Figure 3B).

\section{The inhibitory effect of free-FAs is mediated by attenuated peroxisome-proliferator-activated receptor- $\gamma$ coactivator 1a (PGC1a) response}

The similar effect of FAs upon PEPCK and G6pase expression imply an upstream target for the inhibitory effect, and therefore the effect of free-FAs on PGC1 $\alpha$ induction was examined. 


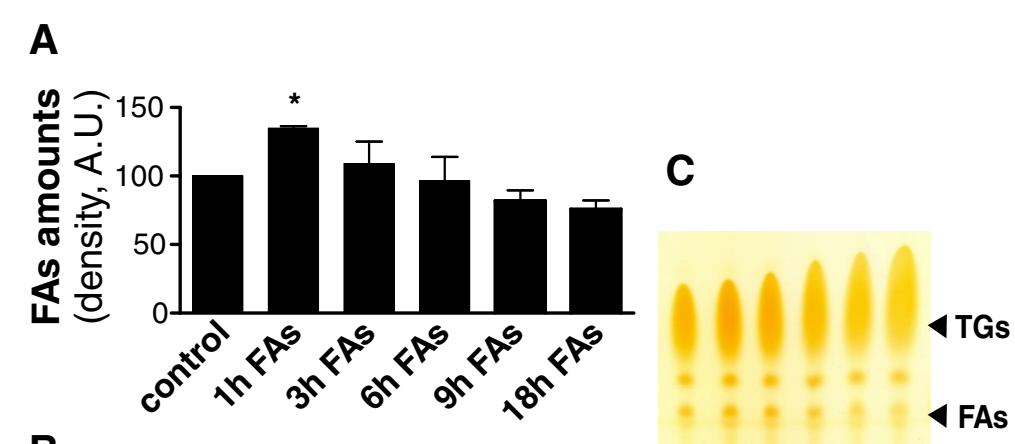

B
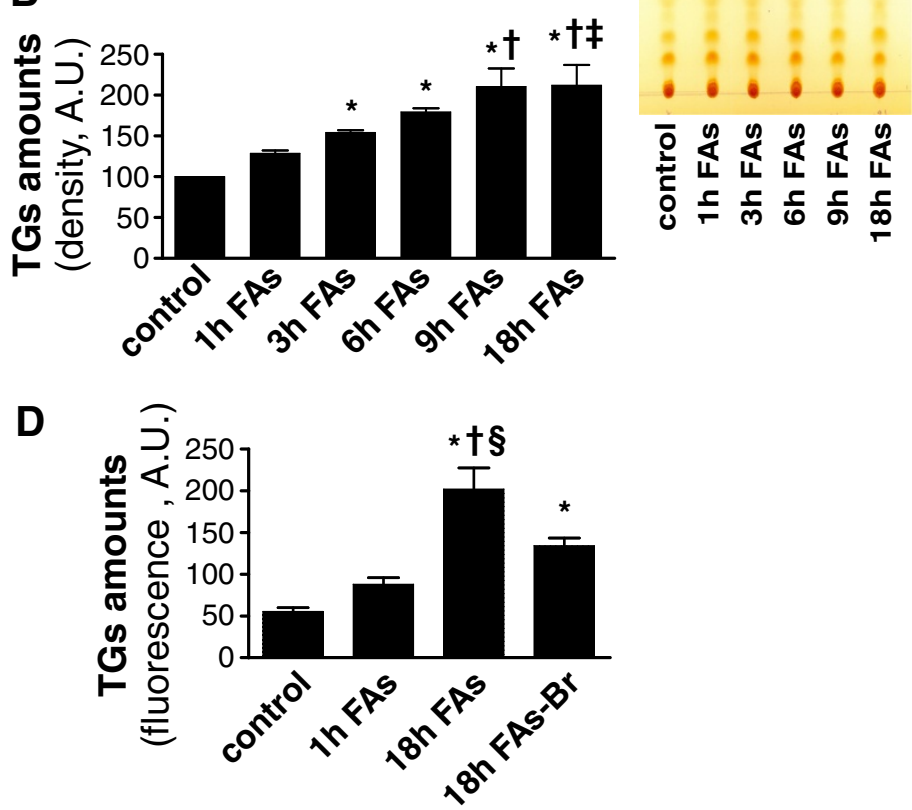

E

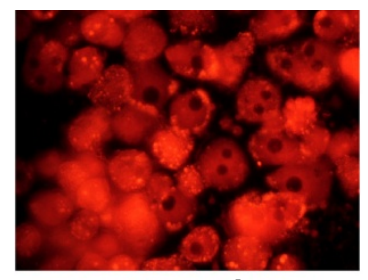

control

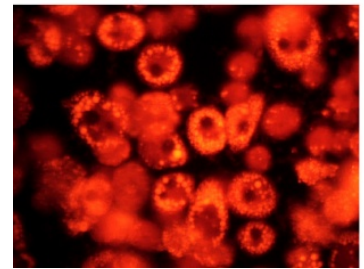

18h FAs

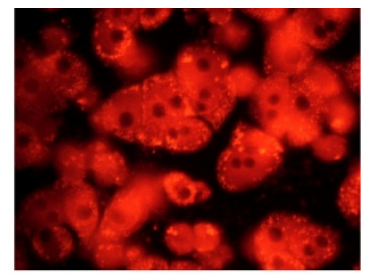

1h FAs

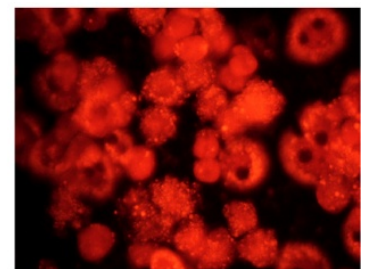

18h FAs-Br

Figure 1 (See legend on next page.)

As shown in Figure 4, pre-treatment with free-FAs for 1 hour results in a prominent inhibition of PGC1 $\alpha$ expression, when induced by glucagon and cAMP (Figure 4A) or by dexamethasone and cAMP (Figure 4B).
In both cases, the effect of free-FAs is diminished when pre-treatment is elongated to 18 hours, but maintained when 18 hours pre-treatment with FAs-Br is issued (Figure $4 \mathrm{~A}$ and $4 \mathrm{~B}$ ). In fact, PGC1 $\alpha$ expression is 


\section{(See figure on previous page.)}

Figure 1 Uptake of free-FAs and accumulation of TGs in FAs-treated cells. Amounts of Free-FAs (A) and TGs (B) were evaluated by thinlayer chromatography in cellular extracts of primary hepatocytes cultured with FAs mixture (2: 1 oleate:palmitate with $1 \%$ BSA) to final concentration of $1 \mathrm{mM}$ for the indicated times. Quantification of lipid spots was performed by scanning densitometry. The bars represent the means \pm SD of the values, relatively to the corresponding control treatment, which was normalized to $100 . n=4$; Comparisons were performed using Tukey's honestly significant differences (HSD) test. ${ }^{*} P<.05$ vs control cells cultured without FAs; $+P<.05$ vs cells cultured with FAs for 1 hour; $¥ P<.05$ vs cells cultured with FAs for 3 hours. (C) A representative silica gel plate separation of cellular lipid extractions is shown, with indication of TGs and free-FAs spots position. (D) Primary hepatocytes were cultured and treated with FAs mixture (2:1 oleate:palmitate with 1\% $\mathrm{BSA}$ ) or with FAs-Br mixture (2:1 oleate:2-Bromopalmitate with 1\% BSA) to final concentration of $1 \mathrm{mM}$ FAs for the indicated times. After that, cells were stained with Nile-Red and fluorescence was measured by FACS. ${ }^{*} P<.05$ vs control cells cultured without FAs; $P<.05$ vs cells cultured with FAs for 1 hour; $\S P<.05$ vs cells cultured with FAs-Br for 18 hours. (E) After the indicated treatments, Nile-Red stained cultures were examined under fluorescence microscopy (magnification $\times 400$ ). Yellow fluorescence indicates intracellular accumulation of TGs.

influenced by the pre-treatments in a similar pattern to PEPCK and G6pase, indicating that PGC1 $\alpha$ can mediate the inhibitory effect of FAs.

\section{Gluconeogenic response attenuation is attributed to decreased CAMP response element-binding (CREB) phosphorylation}

As shown by the evaluation of $\mathrm{p}$-CREB/CREB protein ratio in cellular extracts, CREB activation is induced in response to gluconeogenic stimuli of the cells with glucagon and cAMP (Figure 5A) as well as with dexamethasone and cAMP (Figure 5B). This increase in CREB phosphorylation levels is abolished by pre-treatment of the cells with FAs for 1 hour before gluconeogenic induction, but not by pre-treatment with FAs for 18 hours (Figure 5A and 5B).

Hence, inhibition of CREB activation may precede the observed effects of free-FAs on gluconeogenic response in primary hepatocytes.

\section{Discussion}

Using an in vitro model of primary hepatocytes, this study provided the first evidence that free-FAs can directly attenuate the activation of gluconeogenic pathway by decreasing CREB activation. The results demonstrate that induction of glucose production and gluconeogenic enzymes is strongly inhibited by free-FAs in hepatocytes, and that the mediating link of this inhibitory effect might be inhibition of PGC1 $\alpha$. The inhibitory effect of free-FAs is observed when GNG is induced by different stimuli in hepatocytes, and relieved when they are esterified into TGs. Due to rapid esterification of FAs by the cells, the time window for detection of this effect is found to be short and critical. However, the duration of the effect of free-FAs is extended when their esterification is prevented by using non-metabolic FAs. Together, this implies a mechanism by which GNG is suppressed physiologically when FAs-metabolism takes over during long term fasting in vivo. Furthermore, exceptionally high levels of free-FAs found in the milieu of the $\beta$ - oxidation deficient liver can directly attenuate GNG and therefore facilitate hypoglycemia among patients.

Although a role for FAs in modulation of GNG was previously suggested, it remained controversial whether FAs affect hepatic glucose production and how [11]. Some findings imply that free-FAs increase hepatic GNG in both healthy and diabetic individuals [12,13], as well as in the perfused liver $[14,15]$, and in isolated hepatocytes $[16,17]$. Yet, the mechanism underlies this proposed stimulatory effect is far from being elucidated. Due to correlation between lipid utilization and hepatic GNG rates [16,18], the effect was mostly attributed to increased FAs oxidation. Other data demonstrate that FAs activate mitochondrial transport of pyruvate independently of their oxidation [14], thus rule out that acetyl-CoA and NADH levels are involved. Alternatively, FAs oxidation was suggested to directly stimulate gluconeogenic enzymes [19].

In contrast, an inhibitory effect of FAs on hepatic GNG has also been demonstrated both in vitro [20] and in vivo [21]. Another study reported stimulatory as well as inhibitory effects of FAs on glucose production in hepatocytes, depending upon the type of FAs and the type of gluconeogenic precursor used [22]. Therefore, some of the inconsistency could arise from the diversity of FAs, different exposure times, and variety of experimental models used in various studies. Another explanation to some of controversy might be that FAs activate GNG only in the fed state, but not under fasting [16] or when GNG is induced [23], and may even attenuate GNG during fasting [24].

Our findings, indicating an inhibitory effect of FAs on PEPCK expression in hepatocytes, are seemingly not compatible with several former data. Those include studies reporting stimulation of PEPCK expression by short-chain FAs in hepatoma cells and in primary hepatocytes, as well as by mid-, and long-chain FAs in primary hepatocytes $[17,25]$. Stimulatory effect was also demonstrated by oleate in FaO hepatoma cell line [26], although later experiments have failed to reproduce this result in other hepatoma cell lines or in primary hepatocytes (reported in [27]). Anyhow, 


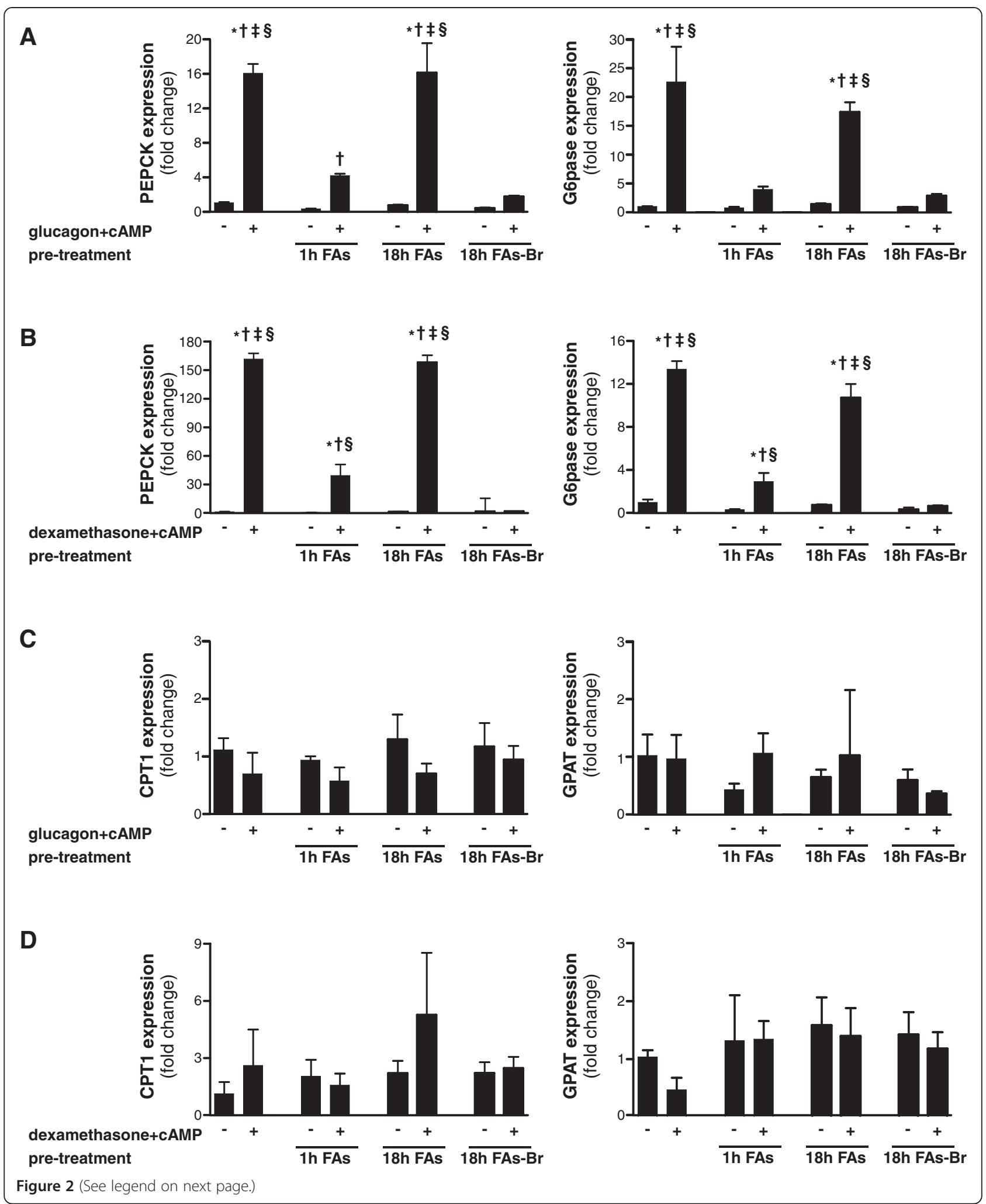

performed in absence of gluconeogenic stimulus, all these previous studies reflect the effect of FAs on basal PEPCK expression rather than the effect under inductive conditions which was examined in the current study. The current data indicate that free-FAs robustly attenuate the gluconeogenic stimuli-induced PEPCK expression in 

cultured and pre-treated with FAs mixture (2:1 oleate:palmitate with 1\% BSA) or with FAs-Br mixture (2:1 oleate:2-Bromopalmitate with 1\% $\mathrm{BSA}$ ) to final concentration of $1 \mathrm{mM}$ FAs for the indicated times. After pre-treatments, cells were treated with the gluconeogenic inducers glucagon and CAMP (A and $\mathbf{C}$ ) or dexamethasone and CAMP (B and $\mathbf{D})$ for 6 hours. mRNA expression levels of phosphoenolpyruvate carboxykinase (PEPCK), glucose-6-phosphatase (G6pase), carnitine palmitoyltransferase 1 (CPT1), and glycerol-3-phosphate acyltransferase (GPAT) were measured by quantitative real-time RT-PCR. Data are expressed as means \pm SD, presented relatively to basal expression level (of cells not pre-treated with FAs nor treated with gluconeogenic inducers), which was normalized to 1. $n=3$; Comparisons were performed using Tukey's honestly significant differences (HSD) test. ${ }^{*} P<.05$ vs basal expression level; $\uparrow P<.05$ vs respective control treatment (of cells not treated with gluconeogenic inducers); $\$ P<.05$ vs cells pre-treated for 1 hour with FAs and then treated with gluconeogenic inducers; $\S P<.05$ vs cells pre-treated for 18 hours with FAs-Br and then treated with gluconeogenic inducers.

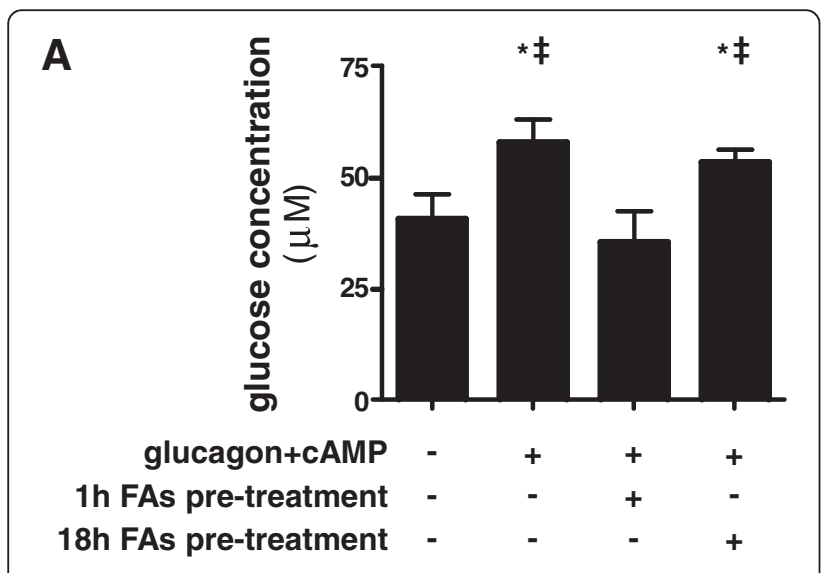

B
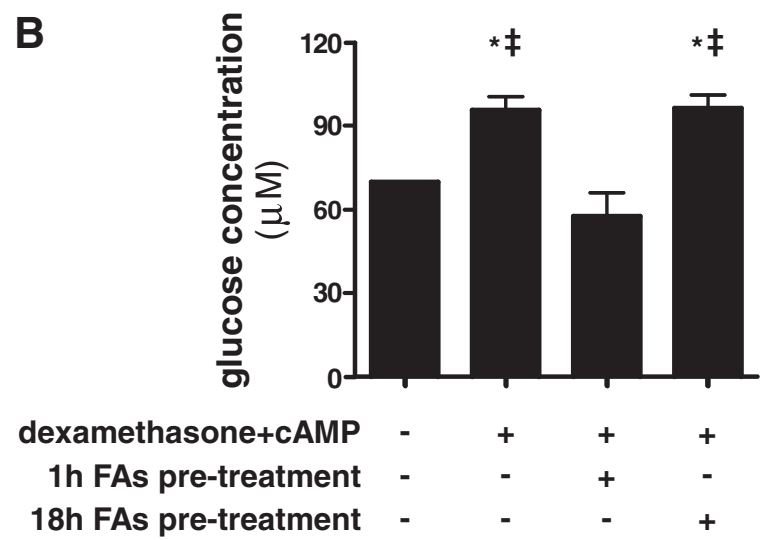

Figure 3 Inhibition of induced glucose release by FAs. Primary hepatocytes were cultured and pre-treated with FAs mixture (2: 1 oleate: palmitate with $1 \% \mathrm{BSA}$ ) to final concentration of $1 \mathrm{mM}$ FAs for the indicated times. After pre-treatments, cells were treated with the gluconeogenic inducers glucagon and CAMP (A) or dexamethasone and cAMP (B) for 6 hours. Glucose output was determined by glucose concentration measured in medium, which initially contained gluconeogenic precursors but no glucose. Glucose levels were normalized per million cells and are expressed as means $\pm S D$. $n=3$; Comparisons were performed using Tukey's honestly significant differences (HSD) test. ${ }^{*} P<.05$ vs basal output level; $\sharp P<.05$ vs cells pre-treated for 1 hour with FAs and then treated with gluconeogenic inducers. hepatocytes. Since there is no known post-translational regulation of PEPCK [27], inhibition of gene induction is expected to be directly reflected in reduction of enzyme activity.

G6pase was also previously investigated by a number of studies as an optional site for FAs involvement in GNG regulation, yet much discrepancy exists between different in vitro and in vivo findings of FAs effect on G6pase expression [28]. Stimulatory effect upon G6pase expression was reported by short-chain FAs in primary hepatocytes and hepatoma cell cultures, and by midand long-chain FAs in isolated hepatocytes [17,29]. Another study found no effect of saturated and monounsaturated FAs, but a suppression effect of G6pase expression by polyunsaturated FAs in hepatoma cells [30]. Yet again, performed in a hormone-free environment, these studies are not representative of FAs effect when GNG is physiologically induced in vivo.

It should be noted that, as seen in Figure 2, free-FAs also create a small, not significant, decrease in basal PEPCK and G6pase expression (of cells not stimulated with gluconeogenic inducers). Still, the physiological importance of this observation is unclear, since basal expression levels are very low relatively to the induced expression levels. An inhibitory effect of FAs under conditions of GNG activation is in line with findings indicating suppressive effect of FAs on GNG in the fasting state in vivo [24].

Since FAs are metabolized by hepatocytes, their effect could be attributed to FAs per se, as well as to various FAs-derived metabolites, acting as a relay. Yet, the rapid effect demonstrated, obtained after 1 hour pre-treatment with FAs, does not support the idea of metabolitemediated effect. In addition, the inhibitory effect obtained using FAs-Br, containing the nonmetabolized FA 2-bromopalmitate, is no lesser than the inhibition caused by metabolized FAs. 2-bromopalmitate is not a substrate for $\beta$-oxidation or TGs synthesis, and virtually does not incorporated into neutral lipids but rather inhibits FAs metabolism [31]. This implies that the effect is generated by FAs themselves. Nevertheless, a quick indirect effect, mediated by a FAs metabolite(s), cannot be completely excluded. 


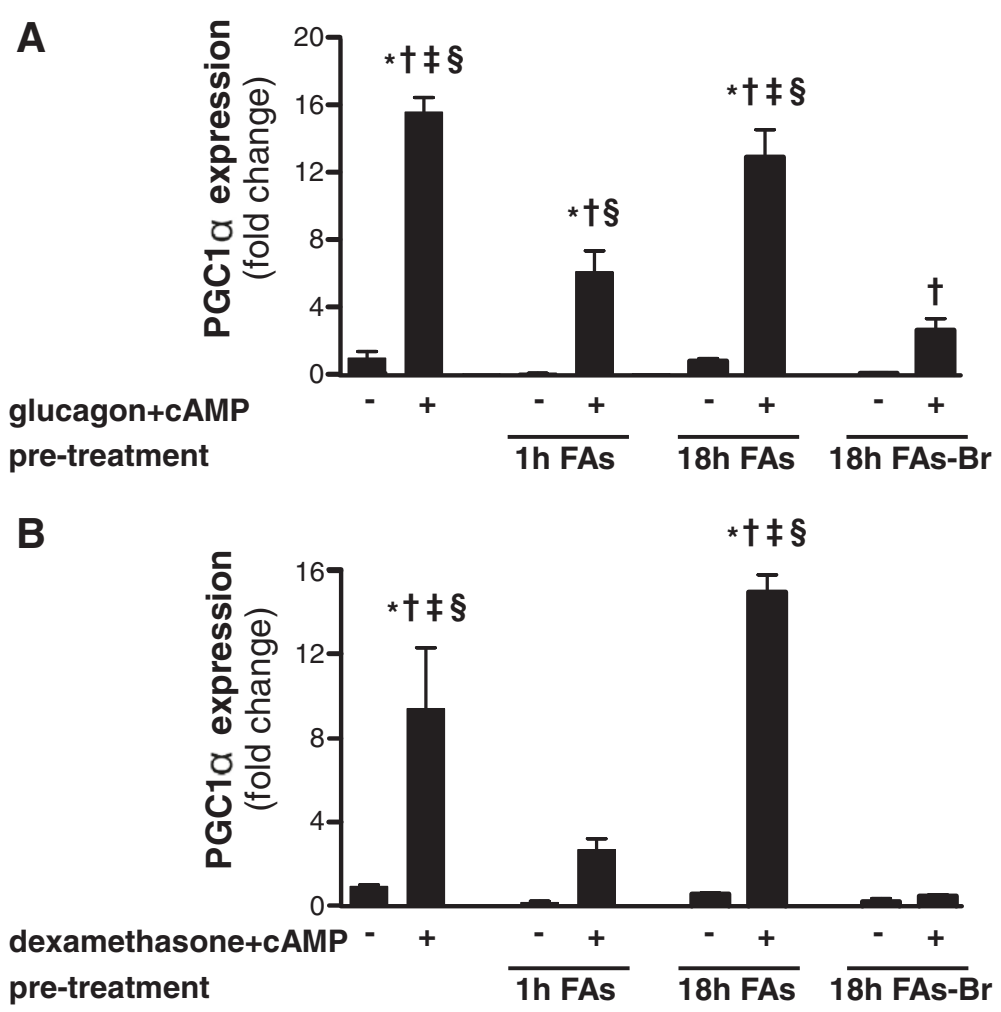

Figure 4 Inhibition of PGC1a gene upregulation by FAs. Primary hepatocytes were cultured and pre-treated with FAs mixture ( $2: 1$ oleate: palmitate with 1\% BSA) or with FAs-Br mixture (2: 1 oleate:2-Bromopalmitate with 1\% BSA) to final concentration of $1 \mathrm{mM} F A s$ for the indicated times. After pre-treatments, cells were treated with the gluconeogenic inducers glucagon and CAMP (A) or dexamethasone and CAMP (B) for 6 hours. mRNA expression levels of PGC1a were measured by quantitative real-time RT-PCR. Data are expressed as means $\pm S D$, presented relatively to basal expression level (of cells not pre-treated with FAs nor treated with gluconeogenic inducers), which was normalized to 1. $n=3$; Comparisons were performed using Tukey's honestly significant differences (HSD) test. ${ }^{*} P<.05$ vs basal expression level; $+P<.05$ vs respective control treatment (of cells not treated with gluconeogenic inducers); $₫ P<.05$ vs cells pre-treated for 1 hour with FAs and then treated with gluconeogenic inducers; $\S P<.05$ vs cells pre-treated for 18 hours with $\mathrm{FAs}-\mathrm{Br}$ and then treated with gluconeogenic inducers.

Unlike key gluconeogenic enzymes, expression levels of main lipid metabolism-related genes CPT1 and GPAT were not affected. These results are in agreement with previous studies indicating that long-chain FAs have no stimulatory effect on CPT1 gene expression in primary hepatocytes [32] and that the response of GPAT gene expression to fasting and refeeding is regulated by insulin [33]. They imply that the inhibitory effect of free-FAs upon the induction of gluconeogenic genes is direct, rather than created as a secondary result to changes induced in lipid metabolism pathways. As both PEPCK and G6pase are rate-limiting enzymes of GNG, inhibition of their induction by free-FAs resulted in elimination of the cellular gluconeogenic response, reflected by glucose release capacity of the cells (presented in Figure 3).

Additional factor that is found to exhibit the same inhibitability toward free-FAs as PEPCK and G6pase genes is $\mathrm{PGC} 1 \alpha$ induction. PGC1 $\alpha$ is a transcriptional coactivator, involved in expression regulation of multiple energy metabolism-related genes in the liver. When induced by gluconeogenic stimuli, PGC1 $\alpha$ activates the entire programme of gluconeogenic genes, including PEPCK and G6pase [34], probably by interaction with HNF-4 and the glucocorticoid receptor [2]. Therefore, FAs can inhibit the induction of gluconeogenic genes by downregulating the $\mathrm{PGC} 1 \alpha$ response.

Finally, the current findings demonstrate that the induced phosphorylation of CREB is inhibited by freeFAs. Activated by phosphorylation at Ser 133, CREB is a transcription factor which binds selectively to the consensus cAMP responsive element (CRE) sequence found in target genes promoters [35,36]. p-CREB induces PGC1 gene trough CRE element found in -130 position to transcription starting point [37]. Moreover, CRE elements are found in both PEPCK [38] and G6pase [39,40] genes and were shown to mediate the cAMP responsiveness of these promoters. Therefore, the decreased CREB activation can inhibit induction of gluconeogenic genes both directly as well as via reduced PGC-1 $1 \alpha$ expression. 


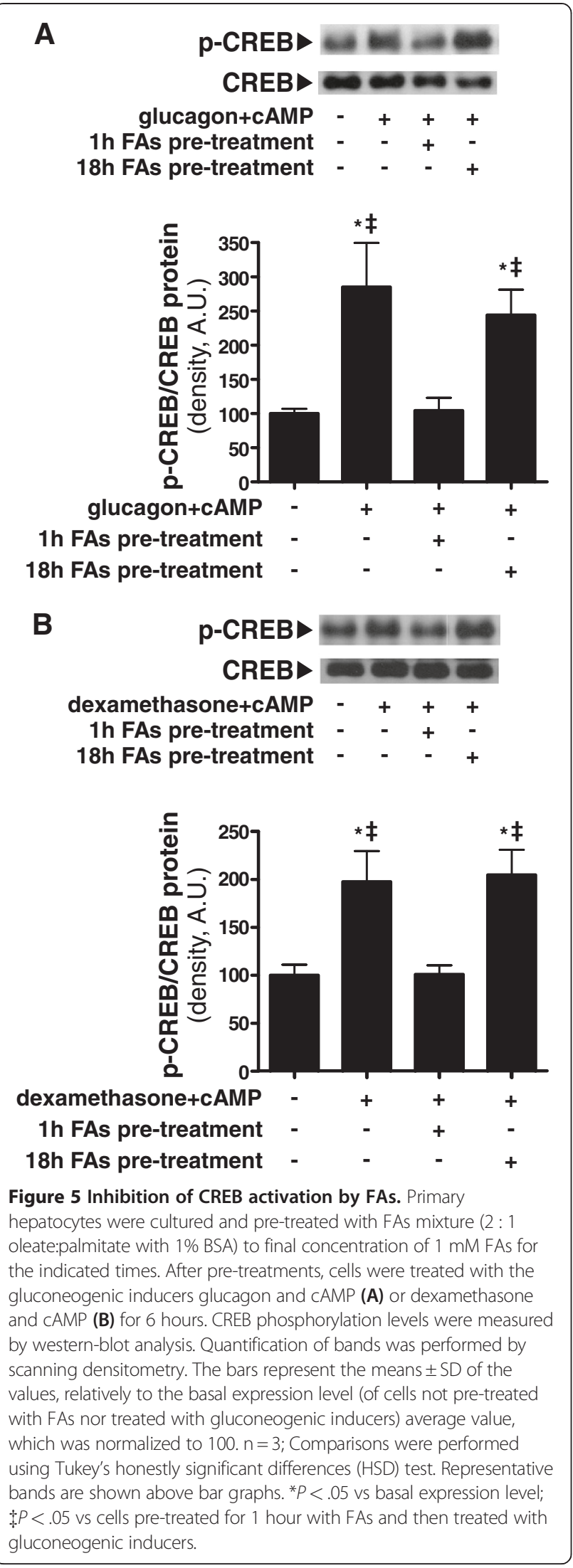

Several transcription-related proteins have been suggested to mediate effects of FAs on genes expression [41], while modification of transcription factors phosphorylation to alternate transactivation capacity is one proposed mechanism [42]. Interestingly, FAs were shown to stimulate basal GNG by PGC- $1 \alpha$ and CREB in isolated hepatocytes [17], supporting the involvement of these activators in the inhibitory effect upon induced GNG. To our knowledge, the inhibition of CREB by FAs had not been reported hitherto.

\section{Conclusions}

In summary, the presented results identify a critical role for free-FAs as inhibitors of induced GNG, which is mediated by influencing PGC1 $\alpha$ and CREB-related signaling. By directly attenuating gluconeogenic activation in the $\beta$-oxidation deficient liver, high levels of free-FAs can promote hypoglycemia. This hypothesis suggests a novel mechanism that underlies this metabolic aberration.

\section{Methods}

Animals

Rats were purchased from Harlan Laboratories and were kept under standard conditions with 12 hours light/dark cycles and free access to food and water. All procedures were performed in accordance with the institutions' guidelines of animal care.

\section{Primary hepatocytes isolation and culturing}

Hepatocytes were isolated according to the method described [43], with minor modifications. Briefly, after anesthesia, rat livers were perfused with Hanks' balanced salt solution (HBSS) containing $1 \mathrm{mM}$ EGTA, followed by perfusion with $0.05 \%$ collagenase (cat.LS004177, Worthington, Lakeswood, NJ) in HBSS in a recirculating matter. The liver was then detached and filtered through a $70 \mu \mathrm{m}$ nylon mesh and cells were sedimented by centrifugation. Cells were plated onto six-well plates $(700,000$ cells $/ \mathrm{ml})$ and grown in low glucose Dulbecco's modified Eagle's medium (DMEM) supplemented with $10 \%$ fetal calf serum, $1 \% \mathrm{~L}$-glutamine, $100 \mu \mathrm{g} / \mathrm{ml}$ penicillin, and $100 \mu \mathrm{g} / \mathrm{ml}$ streptomycin, when kept at $37^{\circ} \mathrm{C}$ in humidified atmosphere (95\% air and $5 \% \mathrm{CO}_{2}$ ).

\section{Preparation of FAs}

Bovine serum albumin (BSA)-complexed FAs were prepared as described [44]. FAs were dissolved in ethanol at a concentration of $100 \mathrm{mM}$. Then BSA solution $(20 \%$, $\mathrm{w} / \mathrm{v}$ ) was heated to $45^{\circ} \mathrm{C}$, and FAs solution was gradually added to the BSA solution to achieve a complexed FAs stock solution (8 $\mathrm{mM})$. 
Induction of FAs stress and GNG in primary hepatocytes 4-6 hours after plating, culture medium was replaced and cells were pre-treated with $1 \mathrm{mM}$ FAs mixture of BSA-complexed palmitic acid and oleic acid (1:2) for up to 18 hours [45]. In some experiments, BSA-complexed palmitic acid in the mixture was replaced with BSAcomplexed 2-bromopalmitate (FAs-Br treatment). Control cells were treated with appropriate vehicle solution (composed of BSA and ethanol).

Gluconeogenic stimuli was induced by $0.1 \mu \mathrm{M}$ glucagon (cat.G2044, Sigma), or $1 \mu \mathrm{M}$ dexamethasone (cat. D4902, Sigma), and $1 \mu$ M Bt2cAMP (cat.D0627, Sigma) added to the culture medium for 6 hours $[34,46]$.

Detection of intracellular triglycerides (TGs) accumulation Nile-Red (cat.N3010, Sigma) stock solution (1 mg/mL) was prepared in dimethyl sulfur oxide and kept at $-20^{\circ}$ C. Cells were washed with phosphate-buffered saline (PBS), $\mathrm{pH} 7.4$, and then incubated for 10 minutes in $37^{\circ}$ $\mathrm{C}$ with Nile-Red solution, freshly prepared in PBS at a concentration of $1 \mu \mathrm{g} / \mathrm{mL}$. Next, cells were washed again with PBS and examined by fluorescence microscopy with excitation at $540 \mathrm{~nm}$ and emission at $605 \mathrm{~nm}$ (Eclipse TS100 with Epi-fluorescence Attachment; Nikon, Tokyo, Japan) and measured by flow cytometry with excitation at $488 \mathrm{~nm}$ and emission at $575 \mathrm{~nm}$ (FACSCalibur; Becton Dickinson, CA, USA)

\section{Extraction of lipids from cells}

Cultured cells were scraped, centrifuged, resuspended in PBS, and sampled for determination of protein concentration by Bradford method (cat.B6916, Sigma). Cells were centrifuged again, resuspended in chloroform:methanol (2:1), and shacked gently for 30 minutes. Distilled water (20\%, v/v) was added and phases separation was accomplished by 15 minutes incubation at room temperature, followed by centrifugation for $15 \mathrm{~min}$ at $470 \times \mathrm{g}\left(4^{\circ} \mathrm{C}\right)$. Chloroform phase containing lipids was collected and vacuum-dried. Pellets were redissolved in chloroform for further analysis.

\section{Thin-layer chromatography (TLC)}

Lipid extraction samples were analyzed relative to standards on a silica gel plate (Merck, Germany), as described [47]. The solvent system comprised petrol ether, diethyl ether, and acetic acid in a volumetric ratio of $80: 19: 1$. Visualization of the compounds on the plates was performed with iodine staining. Quantitative determination of lipid spots intensity was performed by scanning densitometry.

\section{Total RNA isolation and quantitative real-time RT-PCR} analysis

Total RNA was isolated from the cells by using Trizol according to the instructions of the manufacturer. cDNA was generated by reverse transcription of $1 \mu \mathrm{g}$ of total RNA using High Capacity cDNA Reverse Transcription Kit (cat.4368814, Applied Biosystems, Foster City, CA). The expression level of mRNA was quantified with realtime PCR by using Sybr Green qPCR mix in a 7300 Real-Time PCR System (Applied Biosystems, Foster City, CA). The results were normalized to the Glyceraldehyde 3-phosphate dehydrogenase (GAPDH) expression (used as endogenous control) and fold-change expression was calculated by using $\mathrm{Ct}$ values in comparison with experimental controls that received a value of 1 . Primer sequences used are reported in Table 1.

\section{Glucose production assay}

Culture medium was replaced with $0.4 \mathrm{ml}$ of glucose production medium consisting of glucose-free DMEM ( $\mathrm{pH}$ 7.4), without phenol red, supplemented with $20 \mathrm{mM}$ sodium lactate and $2 \mathrm{mM}$ sodium pyruvate [34]. After 1 hour the buffer was collected and the glucose concentration was measured by enzymatic colorimetric glucose assay.

\section{Western blot analysis}

Cells were scraped and lysed on ice in $100 \mu \mathrm{l}$ ice-cold RIPA buffer [50 mM Tris- $\mathrm{HCl}, \mathrm{pH}$ 7.4, $150 \mathrm{mM} \mathrm{NaCl}, 4 \mathrm{mM}$ EDTA, 1\% triton-X, $0.1 \%$ SDS, $1 \%$ sodium deoxycholate, $2 \mathrm{mM}$ sodium orthovanadate, $5 \mathrm{mM} \mathrm{NaF}$ ] with freshly added $1 \mathrm{mM}$ PMSF and protease inhibitor cocktail (cat. P8340, Sigma). Lysates were transferred through 23 G needles, incubated on ice for 30 minutes, vigorously vortexed and centrifuged for $15 \mathrm{~min}$ at $12000 \times \mathrm{g}\left(4^{\circ} \mathrm{C}\right)$. Supernatants were collected and used for Western blot analysis.

Determination of total protein concentrations was carried out by the DC Protein Assay Reagents (cat.5000116, Biorad, Hercules, CA). Protein expression was analyzed by standard Western blot techniques. In brief, protein samples were denatured in electrophoresis buffer

\section{Table 1 Primers used for real-time PCR}

\begin{tabular}{ll}
\hline Primer name & Oligonucleotide sequence $\mathbf{( 5}^{\prime} \mathbf{- 3}^{\mathbf{\prime}} \mathbf{)}$ \\
\hline PEPCK for. & ACGTGGCTGAGACAAGTGAT \\
PEPCK rev. & GGAAGTGATGGTGACTCCTG \\
G6pase for. & ATGTCTACCCGGCTCAGTT \\
G6pase rev. & CACGGGCTGGTCTATCATTA \\
CPT1 for. & CTGCTGTATCGTCGCACATT \\
CPT1 rev. & GAATGGTGGATCCCAGAAGA \\
GPAT for. & AACGCTGAAATGGAGGAGA \\
GPAT rev. & TCTGAGGCGTGCATGAATAG \\
PGC1a for. & GCTATGAAGCCAATGAGCAC \\
PGC1a rev. & TCAGACTCCCGCTTCTCATA \\
GAPDH for. & ATGATTCTACCCACGGCAAG \\
GAPDH rev. & CTGGAAGATGGTGATGGGTT \\
\hline
\end{tabular}


(cat.S3401, Sigma), at $95^{\circ} \mathrm{C}$ for 5 minutes and equal amounts of protein were subjected to sodium dodecyl sulfate-polyacrylamide gel electrophoresis (SDS-PAGE). Proteins were transferred onto nitrocellulose membranes, and equal loading was confirmed by ponceau red staining. The membranes were blocked in Tris-buffered saline with $0.1 \%$ Tween 20 (TBS-T) containing 5\% nonfat dry milk, or 5\% BSA (in the case of CREB and pCREB, accordingly) for 1 hour at room temperature. Immunodetection was done by using anti-CREB antibody (cat.9197, Cell Signaling, Danvers, MA) or anti-pCREB antibody (cat.9198, Cell Signaling, Danvers, MA), diluted $1: 1000$ in TBS-T with $5 \%$ milk or 5\% BSA, accordingly, at $4^{\circ} \mathrm{C}$ overnight. After a subsequent washing step, peroxidase-conjugated anti-rabbit immunoglobulin (cat.111-035-003, Jackson laboratories, West Grove, PA) was used as a secondary antibody (diluted $1: 3500$ in TBS-T with $2.5 \%$ milk at room temperature for 1 hour). Visualization of immunoreactive bands was performed by using ECL detection reagents, and the signal was detected by short exposure to x-ray film. Bands were quantified by scanning densitometry and expressed as arbitrary units. Expression levels of p-CREB in each sample were normalized to their respective CREB levels.

\section{Statistical analysis}

The results are the means \pm S.D of experiments performed in triplicate. Each experiment was repeated in cells of at least two independent cell isolation procedures. Analysis was performed by ANOVA using JMP Software (SAS Institute, Cary, NC, USA). Comparisons between groups were performed using Tukey's honestly significant differences (HSD) test. Differences with a value of $\mathrm{P}<0.05$ were considered to be statistically significant.

\section{Abbreviations \\ FAs: Fatty acids; GNG: Gluconeogenesis; BSA: Bovine serum albumin; TLC: Thin-layer chromatography; GAPDH: Glyceraldehyde 3-phosphate dehydrogenase; TGs: Triglycerides; PEPCK: Phosphoenolpyruvate carboxykinase; G6pase: Glucose-6-phosphatase; CPT1: Carnitine palmitoyltransferase 1; GPAT: Glycerol-3-phosphate acyltransferase; PGC1 a: Peroxisome-proliferator-activated receptor- $\gamma$ coactivator $1 a$; CREB: CAMP response element-binding.}

\section{Competing interests}

The authors declare that there are no competing interests.

\section{Acknowledgements}

This study was supported by a grant no. 377/06 from the Israel Science Foundation to OT and ZM

\section{Authors' contributions}

OT and ZM conceived the experimental design and performed the proofreading of manuscript. NBH performed the experiments and the statistical analysis and wrote the manuscript. SA conducted the FACS analysis. All authors discussed analyses and interpretation, read and approved the final manuscript.
Received: 5 January 2012 Accepted: 7 June 2012

Published: 7 June 2012

\section{References}

1. Postic C, Dentin R, Girard J: Role of the liver in the control of carbohydrate and lipid homeostasis. Diabetes Metab 2004, 30:398-408.

2. Barthel A, Schmoll D: Novel concepts in insulin regulation of hepatic gluconeogenesis. Am J Physiol Endocrinol Metab 2003, 285:E685-E692.

3. Bennett MJ: Pathophysiology of fatty acid oxidation disorders. J Inherit Metab Dis 2010, 33:533-537.

4. Eaton S: Control of mitochondrial beta-oxidation flux. Prog Lipid Res 2002, 41:197-239.

5. Kompare M, Rizzo WB: Mitochondrial fatty-acid oxidation disorders. Semin Pediatr Neurol 2008, 15:140-149.

6. Spiekerkoetter U, Wood PA: Mitochondrial fatty acid oxidation disorders: pathophysiological studies in mouse models. J Inherit Metab Dis 2010, 33:539-546.

7. Houten SM, Wanders RJ: A general introduction to the biochemistry of mitochondrial fatty acid beta-oxidation. J Inherit Metab Dis 2010, 33:469-477.

8. Sherratt HSA: Hypoglycin, the Famous Toxin of the Unripe Jamaican Ackee Fruit. Trends Pharmacol Sci 1986, 7:186-191.

9. Fromenty B, Pessayre D: Inhibition of mitochondrial beta-oxidation as a mechanism of hepatotoxicity. Pharmacol Ther 1995, 67:101-154.

10. Parker SM, Moore PC, Johnson LM, Poitout V: Palmitate potentiation of glucose-induced insulin release: a study using 2-bromopalmitate. Metabolism 2003, 52:1367-1371.

11. Lam TK, Carpentier A, Lewis GF, van de Werve G, Fantus IG, Giacca A: Mechanisms of the free fatty acid-induced increase in hepatic glucose production. Am J Physiol Endocrinol Metab 2003, 284:E863-E873.

12. Boden G, Chen X, Capulong E, Mozzoli M: Effects of free fatty acids on gluconeogenesis and autoregulation of glucose production in type 2 diabetes. Diabetes 2001, 50:810-816.

13. Chen $X$, lqbal N, Boden $G$ : The effects of free fatty acids on gluconeogenesis and glycogenolysis in normal subjects. J Clin Invest 1999, 103:365-372

14. Gonzalez-Manchon C, Ayuso MS, Parrilla R: Control of hepatic gluconeogenesis: role of fatty acid oxidation. Arch Biochem Biophys 1989, 271:1-9.

15. Williamson JR, Kreisberg RA, Felts PW: Mechanism for the stimulation of gluconeogenesis by fatty acids in perfused rat liver. Proc Natl Acad Sci U S A 1966, 56:247-254.

16. Morand C, Remesy C, Demigne C: Fatty acids are potent modulators of lactate utilization in isolated hepatocytes from fed rats. Am J Physiol 1993, 264:E816-E823.

17. Collins QF, Xiong Y, Lupo EG Jr, Liu HY, Cao W: p38 Mitogen-activated protein kinase mediates free fatty acid-induced gluconeogenesis in hepatocytes. J Biol Chem 2006, 281:24336-24344.

18. Fanelli C, Calderone S, Epifano L, De Vincenzo A, Modarelli F, Pampanelli S, Perriello G, De Feo P, Brunetti P, Gerich JE, et al: Demonstration of a critical role for free fatty acids in mediating counterregulatory stimulation of gluconeogenesis and suppression of glucose utilization in humans. J Clin Invest 1993, 92:1617-1622.

19. Randle PJ, Priestman DA, Mistry SC, Halsall A: Glucose fatty acid interactions and the regulation of glucose disposal. J Cell Biochem 1994, 55(Suppl):1-11.

20. Arinze IJ, Hanson RW: Mitochondrial redox state and the regulation of gluconeogenesis in the isolated, perfused cat liver. FEBS Lett 1973 31:280-282.

21. Wolfe RR, Shaw $\mathrm{JH}$ : Inhibitory effect of plasma free fatty acids on glucose production in the conscious dog. Am J Physiol 1984, 246:E181-E186.

22. Zaleski J, Bryla J: Effects of oleate, palmitate, and octanoate on gluconeogenesis in isolated rabbit liver cells. Arch Biochem Biophys 1977 183:553-562.

23. Exton JH, Corbin JG, Park CR: Control of gluconeogenesis in liver. IV. Differential effects of fatty acids and glucagon on ketogenesis and gluconeogenesis in the perfused rat liver. J Biol Chem 1969, 244:4095-4102.

24. Fery F, Plat L, Melot C, Balasse EO: Role of fat-derived substrates in the regulation of gluconeogenesis during fasting. Am J Physiol 1996, 270:E822-E830. 
25. Massillon D, Arinze IJ, Xu C, Bone F: Regulation of glucose-6-phosphatase gene expression in cultured hepatocytes and H4IIE cells by short-chain fatty acids: role of hepatic nuclear factor-4alpha. J Biol Chem 2003, 278:40694-40701.

26. Antras-Ferry J, Le Bigot G, Robin P, Robin D, Forest C: Stimulation of phosphoenolpyruvate carboxykinase gene expression by fatty acids. Biochem Biophys Res Commun 1994, 203:385-391.

27. Forest C, Franckhauser S, Glorian M, Antras-Ferry J, Robin D, Robin P: Regulation of gene transcription by fatty acids, fibrates and prostaglandins: the phosphoenolpyruvate carboxykinase gene as a model. Prostaglandins Leukot Essent Fatty Acids 1997, 57:47-56.

28. Lam TK, van de Werve G, Giacca A: Free fatty acids increase basal hepatic glucose production and induce hepatic insulin resistance at different sites. Am J Physiol Endocrinol Metab 2003, 284:E281-E290.

29. Chatelain F, Pegorier JP, Minassian C, Bruni N, Tarpin S, Girard J, Mithieux G: Development and regulation of glucose-6-phosphatase gene expression in rat liver, intestine, and kidney: in vivo and in vitro studies in cultured fetal hepatocytes. Diabetes 1998, 47:882-889.

30. Rajas F, Gautier A, Bady I, Montano S, Mithieux G: Polyunsaturated fatty acyl coenzyme A suppress the glucose-6-phosphatase promoter activity by modulating the DNA binding of hepatocyte nuclear factor 4 alpha. J Biol Chem 2002, 277:15736-15744.

31. Oakes ND, Furler SM: Evaluation of free fatty acid metabolism in vivo. Ann N Y Acad Sci 2002, 967:158-175.

32. Louet JF, Chatelain F, Decaux JF, Park EA, Kohl C, Pineau T, Girard J, Pegorier JP: Long-chain fatty acids regulate liver carnitine palmitoyltransferase I gene (L-CPT I) expression through a peroxisome-proliferator-activated receptor alpha (PPARalpha)-independent pathway. Biochem J 2001, 354:189-197.

33. Shin DH, Paulauskis JD, Moustaid N, Sul HS: Transcriptional regulation of p90 with sequence homology to Escherichia coli glycerol-3-phosphate acyltransferase. J Biol Chem 1991, 266:23834-23839.

34. Yoon JC, Puigserver P, Chen G, Donovan J, Wu Z, Rhee J, Adelmant G, Stafford J, Kahn CR, Granner DK, et al: Control of hepatic gluconeogenesis through the transcriptional coactivator PGC-1. Nature 2001, 413:131-138.

35. Mayr B, Montminy M: Transcriptional regulation by the phosphorylationdependent factor CREB. Nat Rev Mol Cell Biol 2001, 2:599-609.

36. Montminy MR, Bilezikjian LM: Binding of a nuclear protein to the cyclicAMP response element of the somatostatin gene. Nature 1987, 328:175-178.

37. Herzig S, Long F, Jhala US, Hedrick S, Quinn R, Bauer A, Rudolph D, Schutz $G$, Yoon $C$, Puigserver $P$, et al: CREB regulates hepatic gluconeogenesis through the coactivator PGC-1. Nature 2001, 413:179-183.

38. Patel YM, Yun JS, Liu J, McGrane MM, Hanson RW: An analysis of regulatory elements in the phosphoenolpyruvate carboxykinase (GTP) gene which are responsible for its tissue-specific expression and metabolic control in transgenic mice. J Biol Chem 1994, 269:5619-5628.

39. Lin B, Morris DW, Chou JY: The role of HNF1alpha, HNF3gamma, and cyclic AMP in glucose-6-phosphatase gene activation. Biochemistry 1997 36:14096-14106

40. Schmoll D, Wasner C, Hinds CJ, Allan BB, Walther R, Burchell A: Identification of a CAMP response element within the glucose- 6phosphatase hydrolytic subunit gene promoter which is involved in the transcriptional regulation by CAMP and glucocorticoids in H4IIE hepatoma cells. Biochem J 1999, 338(Pt 2):457-463.

41. Duplus $E$, Forest $C$ : Is there a single mechanism for fatty acid regulation of gene transcription?. Biochem Pharmacol 2002, 64:893-901.

42. Duplus $\mathrm{E}$, Glorian $\mathrm{M}$, Forest $\mathrm{C}$ : Fatty acid regulation of gene transcription. J Biol Chem 2000, 275:30749-30752.

43. Ilan E, Tirosh O, Madar Z: Triacylglycerol-mediated oxidative stress inhibits nitric oxide production in rat isolated hepatocytes. J Nutr 2005, 135:2090-2095.

44. Aronis A, Madar Z, Tirosh O: Lipotoxic effects of triacylglycerols in $\mathbf{J 7 7 4 . 2}$ macrophages. Nutrition 2008, 24:167-176.

45. Kohli R, Pan X, Malladi P, Wainwright MS, Whitington PF: Mitochondrial reactive oxygen species signal hepatocyte steatosis by regulating the phosphatidylinositol 3-kinase cell survival pathway. J Biol Chem 2007, 282:21327-21336.

46. Louet JF, Hayhurst G, Gonzalez FJ, Girard J, Decaux JF: The coactivator PGC-1 is involved in the regulation of the liver carnitine palmitoyltransferase I gene expression by CAMP in combination with
HNF4 alpha and CAMP-response element-binding protein (CREB). J Biol Chem 2002, 277:37991-38000.

47. Budick-Harmelin N, Dudas J, Demuth J, Madar Z, Ramadori G, Tirosh O: Triglycerides potentiate the inflammatory response in rat Kupffer cells. Antioxid Redox Signal 2008, 10:2009-2022.

doi:10.1186/1476-511X-11-66

Cite this article as: Budick-Harmelin et al:: Fatty acids-stress attenuates gluconeogenesis induction and glucose production in primary hepatocytes. Lipids in Health and Disease 2012 11:66.

\section{Submit your next manuscript to BioMed Central and take full advantage of:}

- Convenient online submission

- Thorough peer review

- No space constraints or color figure charges

- Immediate publication on acceptance

- Inclusion in PubMed, CAS, Scopus and Google Scholar

- Research which is freely available for redistribution 